\title{
Seismic Performance of Regular and Irregular Flat Slab Structure with Soil Structure Interaction
}

\author{
Prateek Pattanashetti and M.S. Bhandiwad
}

\begin{abstract}
Earthquake is a very important aspect to be considered while designing structures. Lot of work has been reported by many researchers who worked to study the effect of structures with irregular plan. This paper presents effects of plan and shape configuration on irregular shaped structures. Buildings with irregular geometry respond differently against seismic action. Plan geometry is the parameter which decides its performance against different loading conditions. The effect of irregularity (plan) on structure have been carried out by using structural analysis software ETABS for three different types of soil considering the effect of soil structure interaction. There are several factors which affect the behaviour of building from which storey drift and lateral displacement play an important role in understanding the behaviour of structure. Results are expressed in form of graphs and bar charts. Based on these conclusions have been presented.
\end{abstract}

Keywords--- Soil Structure Interaction, Seismic Performance, ETABS, Equilvalent Static Method

\section{INTRODUCTION}

$I_{\mathrm{a}}^{\mathrm{N}}$ many structures flat slab type system is being adopted as this system have more advantages over the framed structure i.e., conventional RC framed structures like speed of construction, reduce in floor height in order to meet the architectural and economical demands, simple form work, less loss in energy of cold storage buildings, un obstructed area etc. Flat slab system is also called a beamless slab where RC slabs directly rests on columns. In this system there will be no beam or girder and loads transfers takes from slab to columns and columns to foundations.

Because of recent occurred earthquake losses in a world have resulted in the awarenes of seismic hazards and corresponding loss to built of environment. Effort have been given in order for reasonable estimates, predictions and mitigation of risks associated with potential loss. Generally flat slab system are design in lower seismic zones for gravity loads and because absence of beams, flat slab structural system is more flexible than the traditional slab-beamcolumn frame system for lateral loads. And even an IS code the provision for ductile detailing of flat slab is not given separately. Hence the flat slab systems are more affected under seismic prone zones. For this reason the study

Prateek Pattanashetti, PG Student, Dept.of Civil Engineering, SDM College of Enginering and Technology, Dharwad, India. E-mail:pratiekshetti@gmail.com

M.S. Bhandiwad, Asst. Professor, Dept.of Civil Engineering, SDMCET, Dharwad, India.E-mail:mallucv014@ gmail.com

DOI:10.9756/BIJMMI.8186 of flat slab buildings under seismic loads is important.

\section{A. Regular and Irregular Buildings}

A Regular building is one which has no significant discontinuities as far as in plans or geometry, lateral load resisting system and vertical configuration. But an irregular building is one which has significant discontinuities in plan or geometry, load path, lateral load resisting systems and horizontal and vertical configuration etc

\section{B. Classification of Irregularities}

Table 1: Classification of Irregularities

\begin{tabular}{|l|l|}
\hline 1 . Plan Irregularities & 2. Vertical irregularity \\
\hline $\begin{array}{l}\text { a. Torsion } \\
\text { Irregularity }\end{array}$ & a. Stiffness irregularity \\
\hline $\begin{array}{l}\text { b. Re-Entrant } \\
\text { corners }\end{array}$ & b. Mass irregularity \\
\hline $\begin{array}{l}\text { c. Diaphragm } \\
\text { Discontinuit }\end{array}$ & c. Vertical geometric irregularity \\
\hline $\begin{array}{l}\text { d. Out of plane } \\
\text { offsets }\end{array}$ & $\begin{array}{l}\text { d. In plane discontinuity in vertical } \\
\text { elements }\end{array}$ \\
\hline $\begin{array}{l}\text { e. Non parallel } \\
\text { systems }\end{array}$ & e. Discontinuity in capacity \\
\hline
\end{tabular}

\section{Effects of Soil-Structure Interaction}

The primary issues involved in the phenomenon of soilstructure interaction are the seismic waves propagate through soil during an earthquake a discontinuity in the medium of wave's propagation is encountered at the interface of soil and structural foundations. The change in the material properties leads to scattering, diffraction, reflection and refraction of the seismic waves at the soil-foundation interface their by changing the nature of ground motion at that point. This further leads to slippage across the soil-foundation interface-a nonlinear phenomenon, which is very difficult to analyse. This leads to a subsequent increase in the natural periods of the structural system.Due to the flexibility of the soil the overall time period of the structure will be elongated. If the structure is founded on hard soil then the soil flexibility will not affect the time period of the structure. If the structure is founded on medium or soft soil and if it is a flexible structure then time period of the structure further increased by considering soil flexibility which will cause reduction in spectral acceleration and the base shear. If the structure is a very flexible structure then increase in time period will cause very small reduction in spectral acceleration. 


\section{Objective of the Present Study}

In this study behaviour of regular and plan irregularity of flat slab building under the seismic response with soil structure interaction (SSI) considered.

1. To study the effect of lateral load resisting system for a regular and irregular flat slab structure.

2. To study the performance under regular and irregular flat slab structure under soil structure interaction (SSI).

3. To study the response of structure by equivalent static force method and response spectrum analysis.

4. To study the parameters such as natural time period, base shear, lateral displacement a and storey drift by using Equivalent static force method and response spectrum analysis.

5. To compare the performance regular $\mathrm{RC}$ flat slab structure with different plan irregular RC flat slab structure.

\section{MODELLING}

Modelling is done using ETABS by following steps

- Define material properties

- Define Frame section properties

- Define Area section properties

- Develop the model and assign the joint restraints

- Define load pattern and assign to frame

- Define Load Combinations

- Run the Analysis

A. General Descriptions of Building

- Plan size: 9 x $9 \mathrm{~m}$

- No of story: 20(G+19)

- Story Height: $3 \mathrm{~m}$

- Grade of concrete: M25

- Grade of Steel: Fe415

- Column size: 900 x $900 \mathrm{~mm}$

- Thickness of slab: $200 \mathrm{~mm}$

- Thickness of drop: $450 \mathrm{~mm}$

- Live load: As per IS875-1987 (Part II)

- Typical floor: $3.0 \mathrm{KN} \backslash \mathrm{m}^{2}$

- Roof: $1.5 \mathrm{KN} \backslash \mathrm{m}^{2}$

- $\quad$ Super dead load (Floor finish):

- Typical floor: $2.0 \mathrm{KN} \backslash \mathrm{m}^{2}$

- Roof: $2.5 \mathrm{KN} \backslash \mathrm{m}^{2}$

The following parameters have used in analysis for both ESM and RS method

Table 2: Seismic Parameters

\begin{tabular}{|l|}
\hline Seismic zone: III (Moderate) \\
\hline Soil type: Hard soil (Type I) \\
\hline Medium soil (Type II) \\
\hline Soft soil (Type III) \\
\hline Importance factor (I): 1 \\
\hline Damping: $5 \%$ \\
\hline Response reduction factor (R): 5 \\
\hline
\end{tabular}

B. Modelling of Regular and Irregular Building

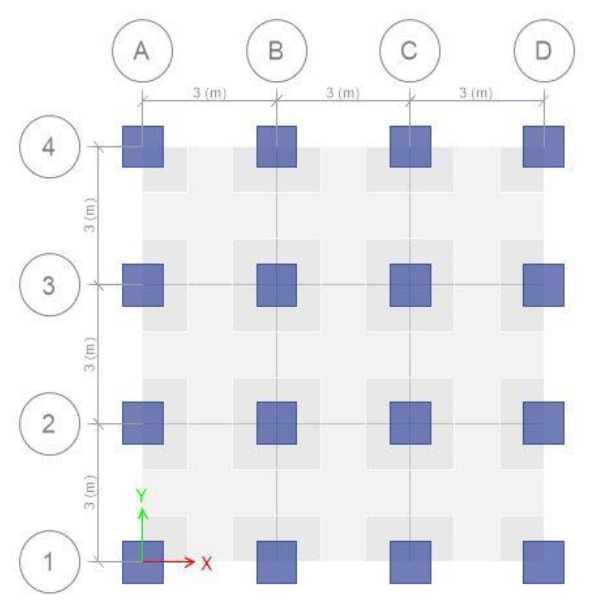

Figure 1: Plan View of Regular Flat Slab Building

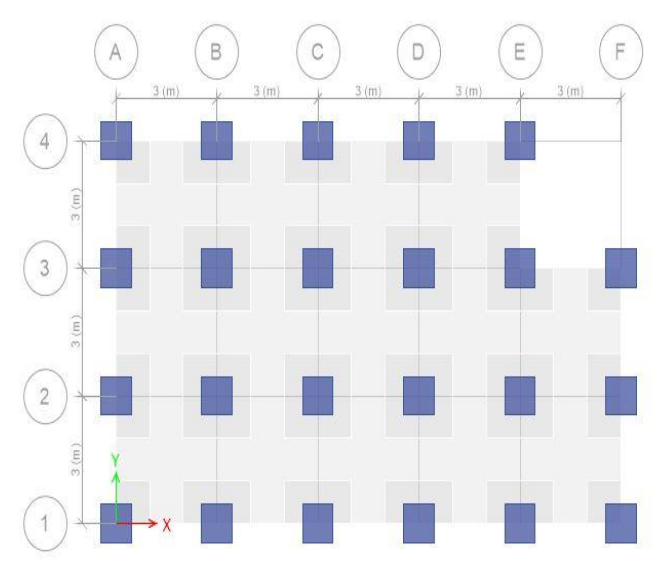

Figure 2: Plan View of Re-entrant Corners Irregularity

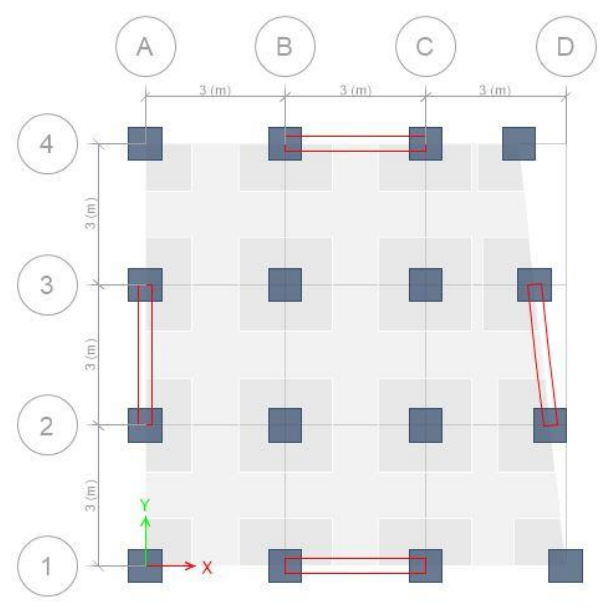

Figure 3: Plan View of Non-Parallel Irregularity Flat Slab Building 


\section{Soil Parameters Considered in the Study}

Table 3: Soil Parameters Considered in the Study

\begin{tabular}{|l|l|l|l|}
\hline Soil type & Hard soil & Medium soil & Soft soil \\
\cline { 1 - 4 } Properties & & & \\
\hline Poisson's ratio, $\mu$ & 0.5 & 0.5 & 0.5 \\
\hline Mass density, $\rho$ & $2000 \mathrm{Kg} / \mathrm{m}^{3}$ & $1700 \mathrm{Kg} / \mathrm{m}^{3}$ & $1500 \mathrm{Kg} / \mathrm{m}^{3}$ \\
\hline Shear wave velocity, $\mathrm{v}$ & $1500 \mathrm{~m} / \mathrm{s}$ & $800 \mathrm{~m} / \mathrm{s}$ & $200 \mathrm{~m} / \mathrm{s}$ \\
\hline Shear modulus, G & 4500000 & 108000 & 60000 \\
& $\mathrm{KN} / \mathrm{m}^{2}$ & $\mathrm{KN} / \mathrm{m}^{2}$ & $\mathrm{KN} / \mathrm{m}^{2}$ \\
\hline Safe Bearing Capacity, & $324 \mathrm{KN} / \mathrm{m}^{2}$ & $245 \mathrm{KN} / \mathrm{m}^{2}$ & $50 \quad \mathrm{KN} / \mathrm{m}^{2}$ \\
SBC & & & \\
\hline
\end{tabular}

\section{RESULTS AND DISCUSSIONS}

The results of all the individual models are being presented in this chapter. The method of analysis included are Equivalent static analysis and Dynamic analysis by Response spectrum method. The graphs are drawn for 20 storeys for a different types of soils. The results obtained for the parameters like Maximum lateral storey displacement, Maximum storey drifts. Out of all load combinations the limit state of collapse results being dominant and the results of such load combinations are displayer in this section

\section{A. Static Analysis Results for Hard soil}

Table 4: Lateral Displacement for Different Plan Irregularities in X-Direction

\begin{tabular}{|l|l|l|l|l|l|}
\hline $\begin{array}{l}\text { Story } \\
\text { Ht }(m)\end{array}$ & Regular & Mass & $\begin{array}{l}\text { Re- } \\
\text { entrant }\end{array}$ & Offsets & $\begin{array}{l}\text { Non- } \\
\text { parallel }\end{array}$ \\
\hline Base & 0.00068 & 0.00068 & 0.00748 & 0.00112 & 0.00695 \\
\hline 3 & 0.3 & 0.3 & 0.3 & 0.3 & 0.2 \\
\hline 6 & 1 & 1 & 1 & 0.9 & 0.5 \\
\hline 9 & 1.8 & 1.8 & 1.8 & 1.7 & 1 \\
\hline 12 & 2.8 & 2.8 & 2.8 & 2.5 & 1.6 \\
\hline 15 & 3.9 & 3.9 & 3.8 & 3.4 & 2.3 \\
\hline 18 & 4.9 & 5 & 4.8 & 4.3 & 3 \\
\hline 21 & 6.1 & 6.1 & 5.9 & 5.2 & 3.8 \\
\hline 24 & 7.2 & 7.2 & 6.9 & 6.1 & 4.6 \\
\hline 27 & 8.3 & 8.3 & 7.9 & 7 & 5.4 \\
\hline 30 & 9.4 & 9.4 & 8.9 & 7.9 & 6.2 \\
\hline 33 & 10.5 & 10.5 & 9.9 & 8.8 & 7 \\
\hline 36 & 11.6 & 11.6 & 10.8 & 9.7 & 7.8 \\
\hline 39 & 12.6 & 12.6 & 11.7 & 10.6 & 8.6 \\
\hline 42 & 13.5 & 13.6 & 12.5 & 11.4 & 9.3 \\
\hline 45 & 14.4 & 14.5 & 13.3 & 12.2 & 10.1 \\
\hline 48 & 15.3 & 15.3 & 14 & 12.9 & 10.8 \\
\hline 51 & 16 & 16.1 & 14.6 & 13.6 & 11.4 \\
\hline 54 & 16.7 & 16.7 & 15.1 & 14.2 & 12 \\
\hline 57 & 17.3 & 17.3 & 15.5 & 14.7 & 12.6 \\
\hline 60 & 17.8 & 17.8 & 15.8 & 15.1 & 13.1 \\
\hline & & & & & \\
\hline
\end{tabular}

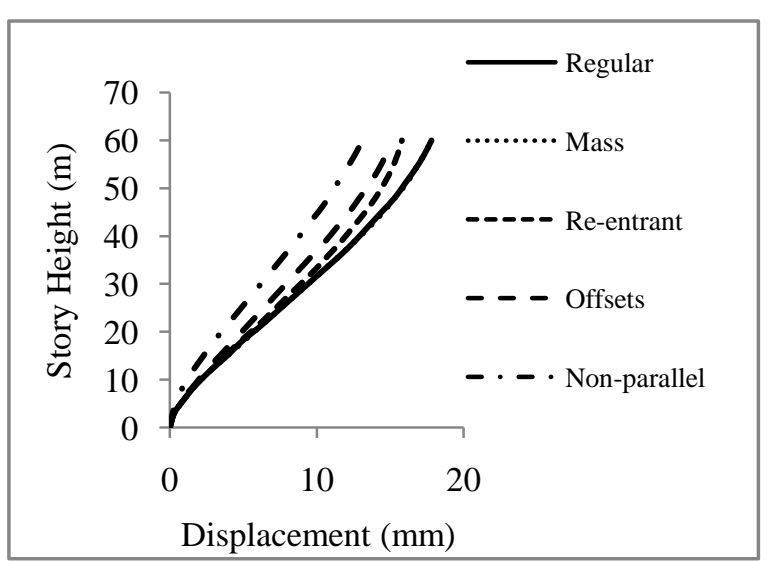

Figure 4: Variations in Lateral Displacement Different Plan Irregularities X-Direction

Table 5: Story Drift for Different Plan Irregularities in XDirection

\begin{tabular}{|l|l|l|l|l|l|}
\hline $\begin{array}{l}\text { Story } \\
\text { Ht(m) }\end{array}$ & Regular & Mass & $\begin{array}{l}\text { Re- } \\
\text { entrant }\end{array}$ & Offsets & $\begin{array}{l}\text { Non- } \\
\text { parallel }\end{array}$ \\
\hline Base & 0 & 0 & 0 & 0 & 0 \\
\hline 3 & 0.294 & 0.297 & 0.306 & 0.309 & 0.183 \\
\hline 6 & 0.669 & 0.669 & 0.675 & 0.621 & 0.363 \\
\hline 9 & 0.867 & 0.87 & 0.861 & 0.756 & 0.492 \\
\hline 12 & 0.981 & 0.984 & 0.957 & 0.825 & 0.594 \\
\hline 15 & 1.047 & 1.05 & 1.005 & 0.864 & 0.666 \\
\hline 18 & 1.086 & 1.089 & 1.029 & 0.891 & 0.723 \\
\hline 21 & 1.11 & 1.11 & 1.035 & 0.909 & 0.762 \\
\hline 24 & 1.119 & 1.122 & 1.032 & 0.918 & 0.789 \\
\hline 27 & 1.119 & 1.122 & 1.02 & 0.921 & 0.807 \\
\hline 30 & 1.11 & 1.11 & 0.999 & 0.918 & 0.813 \\
\hline 33 & 1.089 & 1.092 & 0.969 & 0.906 & 0.828 \\
\hline 36 & 1.059 & 1.062 & 0.93 & 0.885 & 0.804 \\
\hline 39 & 1.02 & 1.02 & 0.882 & 0.858 & 0.783 \\
\hline 42 & 0.969 & 0.969 & 0.825 & 0.822 & 0.756 \\
\hline 45 & 0.909 & 0.909 & 0.756 & 0.777 & 0.726 \\
\hline 48 & 0.837 & 0.837 & 0.678 & 0.723 & 0.687 \\
\hline 51 & 0.756 & 0.759 & 0.591 & 0.663 & 0.648 \\
\hline 54 & 0.672 & 0.672 & 0.501 & 0.594 & 0.609 \\
\hline 57 & 0.588 & 0.588 & 0.411 & 0.522 & 0.57 \\
\hline 60 & 0.519 & 0.519 & 0.363 & 0.462 & 0.54 \\
\hline
\end{tabular}

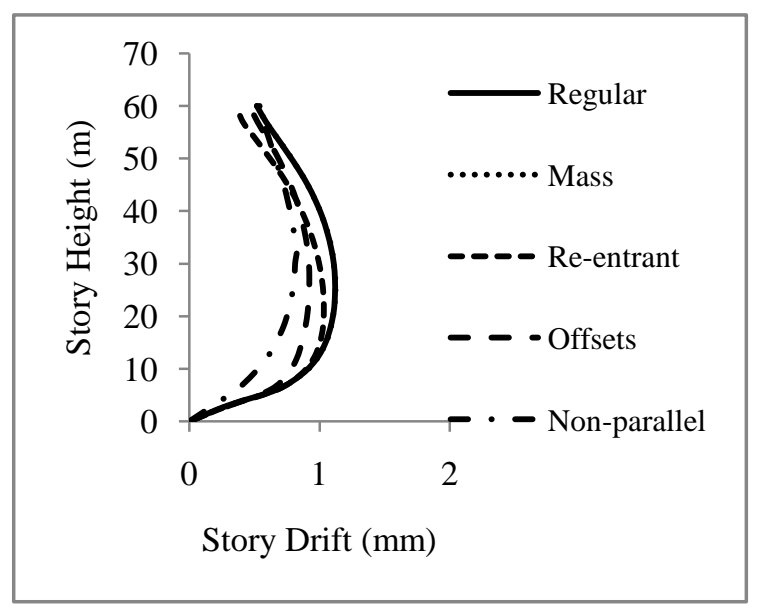

Figure 5: Variations in Storey Drift Different Plan Irregularities X-Direction 


\section{B. Static Analysis Results for Medium Soil}

Table 6: Lateral Displacement for Different Plan Irregularities in X-Direction

\begin{tabular}{|l|l|l|l|l|l|}
\hline Story Ht & Regular & Mass & Re-entrant & Offsets & Non-parallel \\
\hline Base & 0.00192 & 0.00191 & 0.02116 & 0.00315 & 0.01479 \\
\hline 3 & 0.4 & 0.4 & 0.4 & 0.4 & 0.3 \\
\hline 6 & 1.3 & 1.3 & 1.4 & 1.3 & 0.8 \\
\hline 9 & 2.5 & 2.5 & 2.5 & 2.3 & 1.5 \\
\hline 12 & 3.8 & 3.8 & 3.8 & 3.4 & 2.3 \\
\hline 15 & 5.3 & 5.3 & 5.2 & 4.6 & 3.3 \\
\hline 18 & 6.7 & 6.8 & 6.6 & 5.8 & 4.3 \\
\hline 21 & 8.3 & 8.3 & 8 & 7.1 & 5.4 \\
\hline 33 & 14.3 & 14.3 & 13.5 & 12.1 & 9.9 \\
\hline 36 & 15.7 & 15.8 & 14.8 & 13.3 & 11.1 \\
\hline 39 & 17.1 & 17.2 & 16 & 14.4 & 12.2 \\
\hline 42 & 18.5 & 18.5 & 17.2 & 15.6 & 13.3 \\
\hline 45 & 19.7 & 19.7 & 18.2 & 16.6 & 14.3 \\
\hline 48 & 20.8 & 20.9 & 19.1 & 17.6 & 15.3 \\
\hline 51 & 21.9 & 21.9 & 19.9 & 18.5 & 16.3 \\
\hline 54 & 22.8 & 22.8 & 20.6 & 19.3 & 17.1 \\
\hline 57 & 23.6 & 23.6 & 21.2 & 20 & 18 \\
\hline 60 & 24.3 & 24.3 & 21.7 & 20.7 & 18.8 \\
\hline
\end{tabular}

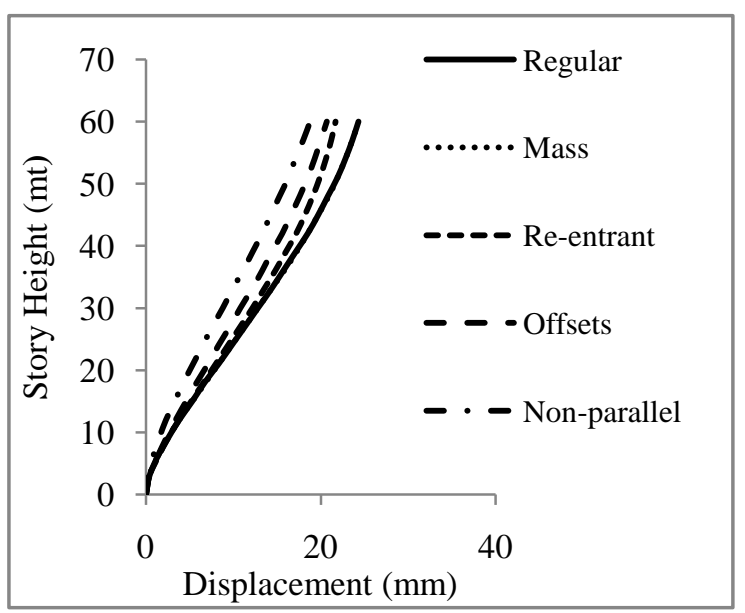

Figure 6: Variations in Lateral Displacement Different Plan Irregularities X-Direction

Table 7: Story Drift for Different Plan Irregularities in XDirection

\begin{tabular}{|l|l|l|l|l|l|}
\hline Story Ht(m) & Regular & Mass & Re-entrant & Offsets & $\begin{array}{l}\text { Non- } \\
\text { parallel }\end{array}$ \\
\hline Base & 0 & 0 & 0 & 0 & 0 \\
\hline 3 & 0.402 & 0.402 & 0.42 & 0.423 & 0.267 \\
\hline 6 & 0.909 & 0.912 & 0.921 & 0.849 & 0.522 \\
\hline 9 & 1.185 & 1.185 & 1.176 & 1.035 & 0.696 \\
\hline 12 & 1.338 & 1.341 & 1.305 & 1.128 & 0.837 \\
\hline 15 & 1.428 & 1.431 & 1.371 & 1.182 & 0.942 \\
\hline 18 & 1.482 & 1.482 & 1.404 & 1.215 & 1.02 \\
\hline 21 & 1.512 & 1.515 & 1.416 & 1.239 & 1.077 \\
\hline 24 & 1.527 & 1.527 & 1.413 & 1.251 & 1.119 \\
\hline 27 & 1.524 & 1.527 & 1.398 & 1.257 & 1.143 \\
\hline 30 & 1.512 & 1.515 & 1.368 & 1.251 & 1.155 \\
\hline 33 & 1.485 & 1.488 & 1.329 & 1.233 & 1.173 \\
\hline 36 & 1.443 & 1.446 & 1.278 & 1.209 & 1.149 \\
\hline 39 & 1.389 & 1.392 & 1.212 & 1.17 & 1.122 \\
\hline 42 & 1.32 & 1.323 & 1.131 & 1.122 & 1.089 \\
\hline 45 & 1.239 & 1.239 & 1.041 & 1.062 & 1.047 \\
\hline 48 & 1.14 & 1.143 & 0.936 & 0.99 & 0.999 \\
\hline 51 & 1.032 & 1.035 & 0.819 & 0.906 & 0.945 \\
\hline 54 & 0.918 & 0.918 & 0.693 & 0.813 & 0.891 \\
\hline 57 & 0.804 & 0.804 & 0.573 & 0.717 & 0.84 \\
\hline 60 & 0.711 & 0.711 & 0.501 & 0.633 & 0.801 \\
\hline & & & & & \\
\hline
\end{tabular}

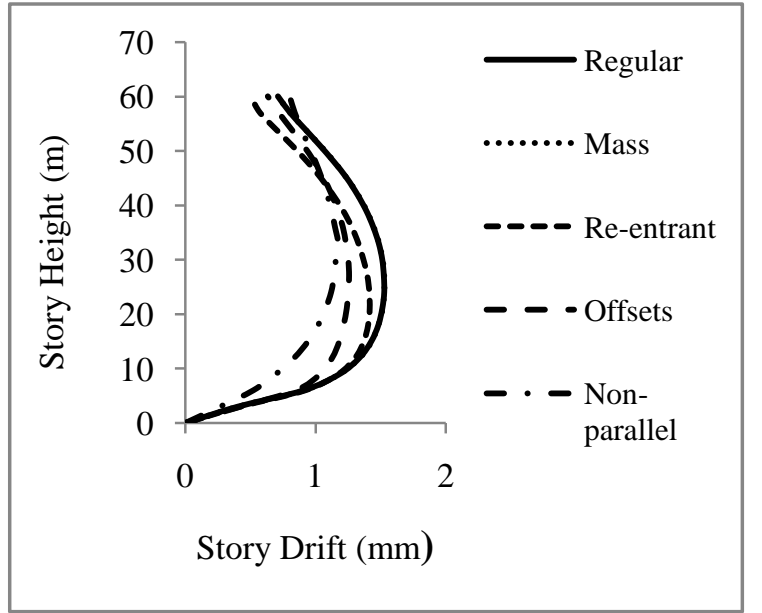

Figure7: Variations in Storey Drift Different Plan Irregularities X-DirectionStatic Analysis Results for Soft soil

Table 8: Lateral Displacement for Different Plan Irregularities in $\mathrm{X}$-direction

\begin{tabular}{|l|l|l|l|l|l|}
\hline $\begin{array}{l}\text { Story } \\
\text { Ht }(m)\end{array}$ & Regular & Mass & $\begin{array}{l}\text { Re- } \\
\text { entrant }\end{array}$ & Offsets & $\begin{array}{l}\text { Non- } \\
\text { parallel }\end{array}$ \\
\hline Base & 0.03251 & 0.02453 & 0.02786 & 0.03796 & 0.1 \\
\hline 3 & 0.6 & 0.5 & 0.6 & 0.6 & 0.5 \\
\hline 6 & 1.8 & 1.7 & 1.7 & 1.8 & 1.3 \\
\hline 9 & 3.3 & 3.2 & 3.2 & 3.1 & 2.3 \\
\hline 12 & 5.1 & 5 & 4.8 & 4.6 & 3.5 \\
\hline 15 & 7 & 6.8 & 6.6 & 6.2 & 4.7 \\
\hline 18 & 8.9 & 8.7 & 8.3 & 7.8 & 6 \\
\hline 21 & 10.9 & 10.6 & 10.1 & 9.4 & 7.4 \\
\hline 24 & 12.8 & 12.6 & 11.9 & 11.1 & 8.9 \\
\hline 27 & 14.8 & 14.5 & 13.6 & 12.7 & 10.3 \\
\hline 42 & 24.2 & 23.7 & 21.6 & 20.6 & 17.6 \\
\hline 45 & 25.8 & 25.3 & 22.9 & 22 & 19 \\
\hline 48 & 27.4 & 26.8 & 24.1 & 23.3 & 20.3 \\
\hline 51 & 28.8 & 28.2 & 25.2 & 24.5 & 21.5 \\
\hline 54 & 30.1 & 29.5 & 26.1 & 25.6 & 22.7 \\
\hline 57 & 31.2 & 30.6 & 26.9 & 26.7 & 23.8 \\
\hline 60 & 32.3 & 31.6 & 27.5 & 27.6 & 24.9 \\
\hline
\end{tabular}

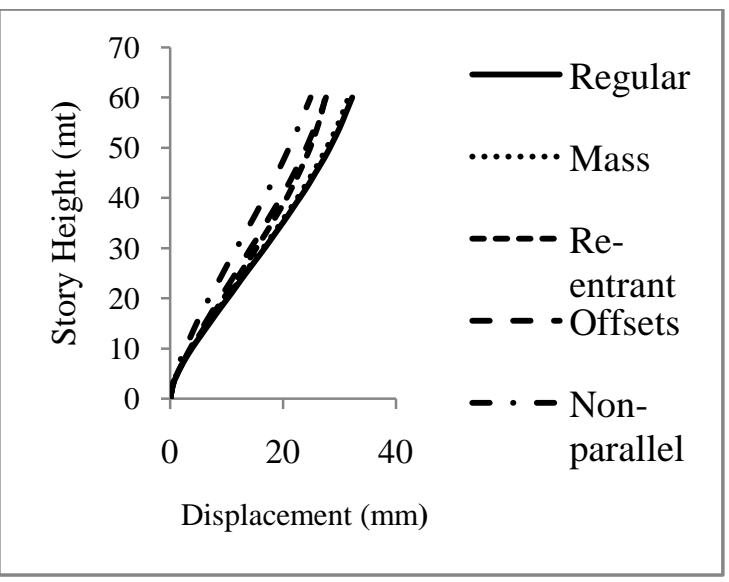

Figure 8: Variations in Lateral Displacement Different Plan Irregularities X-Direction 
Table 9: Storey Drift for Different Plan Irregularities in Xdirection

\begin{tabular}{|l|l|l|l|l|l|}
\hline $\begin{array}{l}\text { Story } \\
\mathrm{Ht}(\mathrm{m})\end{array}$ & Regular & Mass & Re-entrant & Offsets & $\begin{array}{l}\text { Non- } \\
\text { parallel }\end{array}$ \\
\hline Base & 0 & 0 & 0 & 0 & 0 \\
\hline 3 & 0.555 & 0.525 & 0.534 & 0.588 & 0.525 \\
\hline 6 & 1.209 & 1.179 & 1.161 & 1.155 & 0.819 \\
\hline 9 & 1.56 & 1.527 & 1.479 & 1.389 & 0.993 \\
\hline 12 & 1.755 & 1.722 & 1.641 & 1.503 & 1.137 \\
\hline 15 & 1.866 & 1.833 & 1.725 & 1.566 & 1.248 \\
\hline 18 & 1.929 & 1.899 & 1.764 & 1.602 & 1.332 \\
\hline 21 & 1.968 & 1.935 & 1.779 & 1.626 & 1.395 \\
\hline 24 & 1.983 & 1.953 & 1.773 & 1.638 & 1.44 \\
\hline 27 & 1.983 & 1.953 & 1.755 & 1.641 & 1.467 \\
\hline 30 & 1.968 & 1.935 & 1.719 & 1.632 & 1.482 \\
\hline 33 & 1.935 & 1.902 & 1.671 & 1.611 & 1.497 \\
\hline 36 & 1.89 & 1.854 & 1.608 & 1.581 & 1.47 \\
\hline 39 & 1.827 & 1.791 & 1.53 & 1.536 & 1.443 \\
\hline 42 & 1.746 & 1.71 & 1.437 & 1.479 & 1.404 \\
\hline 45 & 1.65 & 1.614 & 1.326 & 1.41 & 1.359 \\
\hline 48 & 1.539 & 1.5 & 1.2 & 1.326 & 1.302 \\
\hline 51 & 1.416 & 1.371 & 1.059 & 1.23 & 1.242 \\
\hline 54 & 1.284 & 1.236 & 0.912 & 1.125 & 1.179 \\
\hline 57 & 1.152 & 1.101 & 0.768 & 1.014 & 1.125 \\
\hline 60 & 1.044 & 0.993 & 0.678 & 0.918 & 1.077 \\
\hline
\end{tabular}

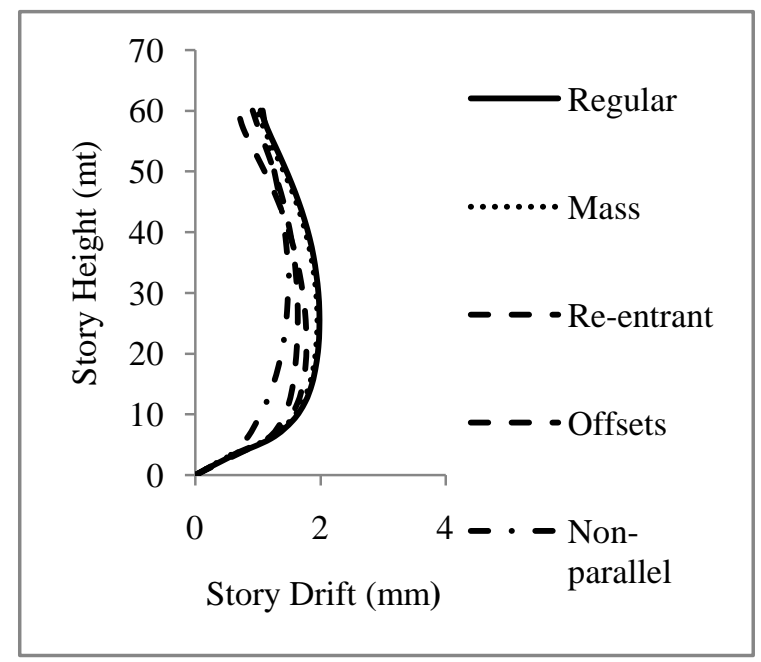

Figure 9: Variations in Storey Drift Different Plan Irregularities X-Direction

\section{CONCLUSION}

\section{For Hard Soil}

1. The maximum and minimum lateral displacements amongst both $\mathrm{X} \& \mathrm{Y}$ direction are $17.8 \mathrm{~mm}$ \& $10.7 \mathrm{~mm}$ for a regular and non-parallel flat slab structure in equivalent static method(ESM) and $13.4 \mathrm{~mm} \& 8.2 \mathrm{~mm}$ for a regular and offsets structure from response spectrum analysis(RSA).

2. The maximum and minimum story drift amongst both $\mathrm{X} \& \mathrm{Y}$ direction are $1.11 \mathrm{~mm} \& 0.83 \mathrm{~mm}$ for a regular and non-parallel flat slab structure in equivalent static method(ESM) and $0.89 \mathrm{~mm} \& 0.53 \mathrm{~mm}$ for a regular and offsets structure from response spectrum analysis(RSA).

\section{For Medium Soil}

1. The maximum and minimum lateral displacements amongst both $\mathrm{X} \& \mathrm{Y}$ direction are $24.3 \mathrm{~mm}$ \& $14.7 \mathrm{~mm}$ for a regular and non-parallel flat slab structure in equivalent static method(ESM) and $18.6 \mathrm{~mm} \& 12.2 \mathrm{~mm}$ for a regular and offsets structure from response spectrum analysis(RSA).

2. The maximum and minimum story drift amongst both $\mathrm{X} \& \mathrm{Y}$ direction are $1.52 \mathrm{~mm} \& 0.95 \mathrm{~mm}$ for a regular and non-parallel flat slab structure in equivalent static method(ESM) and $1.23 \mathrm{~mm} \mathrm{\&} 0.79 \mathrm{~mm}$ for a regular and offsets structure from response spectrum analysis(RSA).

\section{For Soft Soil}

1. The maximum and minimum lateral displacements amongst both X \& Y direction are $32.3 \mathrm{~mm} \& 22.3 \mathrm{~mm}$ for a regular and non-parallel flat slab structure in equivalent static method(ESM) and $25.3 \mathrm{~mm} \&$ $18.7 \mathrm{~mm}$ for a regular and offsets structure from response spectrum analysis(RSA).

2. The maximum and minimum story drift amongst both $\mathrm{X} \& \mathrm{Y}$ direction are $1.98 \mathrm{~mm} \& 1.33 \mathrm{~mm}$ for a regular and non-parallel flat slab structure in equivalent static method(ESM) and $1.62 \mathrm{~mm} \mathrm{\&} 1.09 \mathrm{~mm}$ for a regular and offsets structure from response spectrum analysis(RSA).

\section{REFERENCES}

[1] C.M. Ravikumar, K.S. Babu Narayan, B.V. Sujith and D. Venkat Reddy, "Effect of irregular configuration on seismic vulnerability of RC building", 2012.

[2] Anantwad Shirish, Prof. M.R.Wakchaure and Rohit Nikam, "Effect of Plan Irregularity on High-rise Structures", 2012.

[3] F.A. Syed, B. Dean Kumar, Y. Chandrasekar and B.L.P Swami, "Comparative Analysis of Flat Plate Multistoried Frames With And Without Shear Wall Under Wind Loads", International Journal of Engineering And Advanced Technology, Vol. 2, No. 1, 2012.

[4] G.B. Muralidhara, K.S. Swathi Rani and Mr. Melese Worku, "Seismic Parametric Study on Different Irregular Flat Slab Multi-Story Building" International Journal of Engineering Research \& Technology (IJERT), Vol. 5, No. 4, 2016.

[5] G. Gouramma and Dr.G. Jagadish Kori, "Seismic Performance of Different Rc Slab Systems For Tall Building”, International Journal of Engineering Research, Vol. 3, No. 4, 2015.

[6] K. Venkatarao and N. Nageswarao, "Seismic Behaviour of Reinforced Concrete Framed Structure with Flat and Conventional Floor Slab System", 2016. 